\title{
Design and Analysis of the Bellows Fatigue Test-Bed Based on Electro-Hydraulic Proportional Position Control
}

\section{Entao Zhou ${ }^{1 \mathrm{a}}$, Shenglong Shi ${ }^{2}$, Weiming Wang ${ }^{1}$, Junzhe Lin ${ }^{1}$}

${ }^{1}$ School of Mechanical Engineering and Automation, Northeastern University, China

azhouent@163.com

Keywords: Electro-hydraulic proportional, Dynamic characteristics, PID controller, DSHplus

\begin{abstract}
Electro-hydraulic proportional closed control system is designed which fully integrated electrical and hydraulic advantages of both. The dynamic mathematic model of the hydraulic system is built and the dynamic characteristics of the system are analyzed accurately. The advanced PID controller is adopted to correct the system, and have improved the dynamic quality of the system. In this paper, the hydraulic system is simulated on the basis of the Simulink and DSHplus professional hydraulic simulation software and the results of simulation prove that the qualities of the system can satisfy with the requirement much better.
\end{abstract}

\section{Introduction}

Metal bellows is a flexible, thin wall and a lateral corrugated tubular parts. It basically use its own elastic deformation to work. It is a kind of multi-functional component which is pressureproof, hermetic, antirot, heat resisting and shock-proof except elastic characteristic [1]. International metal bellows appeared in the mid-nineteenth century. The bellows measuring instrument appeared as early as in 1870 [2]. With the further development of science and technology, the further enlargement opening, China's metal bellows technology obtained a rapid development. There's a lot of advanced technology units. For example, Shanghai University of Technology, Nanjing University of Technology, Shenyang Instrument Science Research Institute, Luoyang 725 Research Institute, Nanjing Chenguang Dongluo Bellows Co., LTD, The Capital Spaceflight Metal Bellows Factory and so on [3][4].

\section{The structural design of fatigue test-bed}

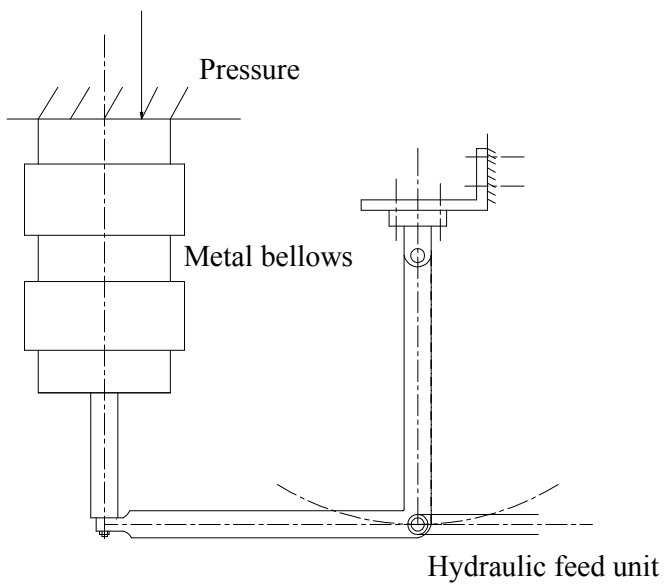

Fig. 1. The simplified diagram of bellows fatigue test mechanism
Testing structure (Fig. 1). The stress of the test is composed of the right hydraulic feed unit and the inner chamber pressure is the creation of the top left unit supply bellows' lumen liquid. In test, the pressure by the right hydraulic feed unit work on the bottom of bellows, it gives bellows a axial force and vacillation to the left and right. The left top inner liquid supply system can control bellows' inner pressure by adjusting flow. This will be a required pressure value, so as to realize test condition of the requirements of the bellows multiaxial fatigue strength. 


\section{Experimental conditions and requirements}

1) Bellows specification is $238 \times 196$, axial stiffness is $21.5 \mathrm{~N} / \mathrm{mm}$, bending stiffness is 11320.42 $\mathrm{N} / \mathrm{mm}$. The axial stiffness hold stable in elastic range, it means that it has a linear relationship between the force and displacement. Bending stiffness is influenced by angle of bending, initial displacement and internal pressure and so on.

2) Bellows internal pressure is $2 \mathrm{MPa}$.

3) On-test, in bellows pressurize process, it must keep the bellows size, it can't have stretch or bend.

4) Oscillating bar swings in sine law way. Amplitude $A=6^{\circ}$, frequency $f=1 \mathrm{~Hz}$. Amplitude $A=0.5^{\circ}$, frequency $f=5 \mathrm{~Hz}$.

5) The allowance of bellows oil-way system must satisfy the test.

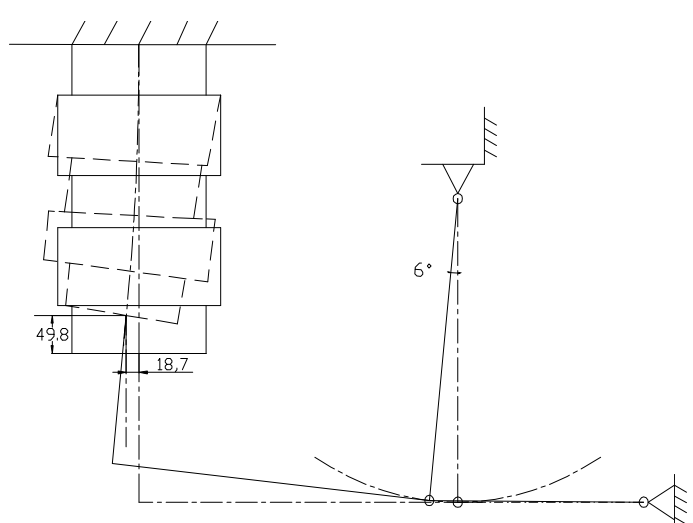

Fig. 2. The state sketch map of bellows Left swing and axial compression

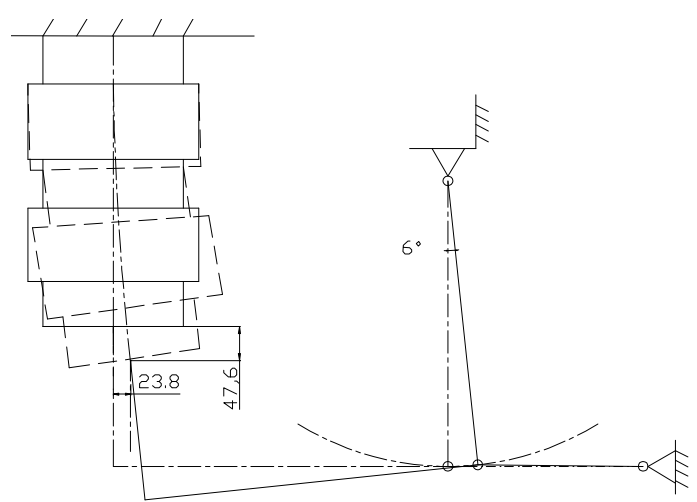

Fig. 3. The state sketch map of bellow right swing and axial extension

On-test, the bellows swinging compression and stretch condition (Fig. 2 and Fig. 3)

\section{The design and calculation of fatigue test-bed hydraulic system}

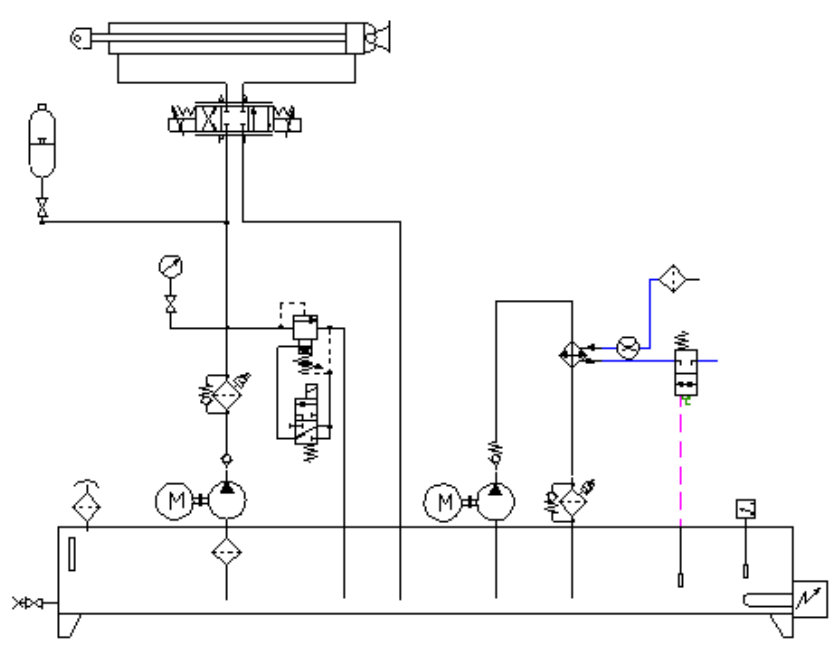

Fig. 4. The principle diagram of fatigue test desk hydraulic system

1) Control system have both open-loop control and closed-loop control, open-loop control don't have antijamming capability. A large number of engineering practice shows that the proportion of open-loop control system's highest position precision can only be $0.3 \mathrm{~mm}$ in existing technology 
condition. And the system need high position precision, so here is the closed-loop system [5].The working process of the fatigue testing, it is known that the closed-loop system is a typical position control system.

2) The schematic diagram of hydraulic system

3) Mathematical model

The transfer function for order $X_{\mathrm{v}}$ input is:

$$
\frac{X_{\mathrm{P}}}{X_{\mathrm{v}}}=\frac{\frac{K_{\mathrm{q}}{ }^{\prime}}{A_{2} \omega_{2}{ }^{\prime}}}{\left(\frac{s}{\omega_{r}{ }^{\prime}}+1\right)\left(\frac{s^{2}}{\omega_{h}{ }^{\prime 2}}+\frac{2 \zeta_{h}{ }^{\prime}}{\omega_{h}{ }^{\prime}} s+1\right)}=\frac{1.87 \times 10^{5}}{\left(\frac{s}{3.85 \times 10^{-4}}+1\right)\left(\frac{s^{2}}{829.29^{2}}+\frac{2 \times 0.2}{829.29} s+1\right)}
$$

The transfer function for interferential order $F_{\mathrm{L}}{ }^{\prime}$ input is:

$$
\frac{X_{\mathrm{P}}}{F_{\mathrm{L}}{ }^{\prime}}=\frac{-\frac{K_{\mathrm{ce}}{ }^{\prime}}{A_{2}{ }^{2} \omega_{2}{ }^{\prime}}\left(1+\frac{V_{\mathrm{t}}{ }^{\prime}}{4 \beta_{\mathrm{e}} K_{\mathrm{ce}}{ }^{\prime}} s\right)}{\left(\frac{s}{\omega_{r}{ }^{\prime}}+1\right)\left(\frac{s^{2}}{\omega_{h}{ }^{\prime 2}}+\frac{2 \zeta_{h}{ }^{\prime}}{\omega_{h}{ }^{\prime}} s+1\right)}=-\frac{7.2 \times 10^{-6} s+2.63 \times 10^{-5}}{\left(\frac{s}{3.85 \times 10^{-4}}+1\right)\left(\frac{s^{2}}{829.29^{2}}+\frac{2 \times 0.2}{829.29} s+1\right)}
$$

\section{The analysis of PID control based on Simulink}

Build closed-loop system simulation model with Simulink software.

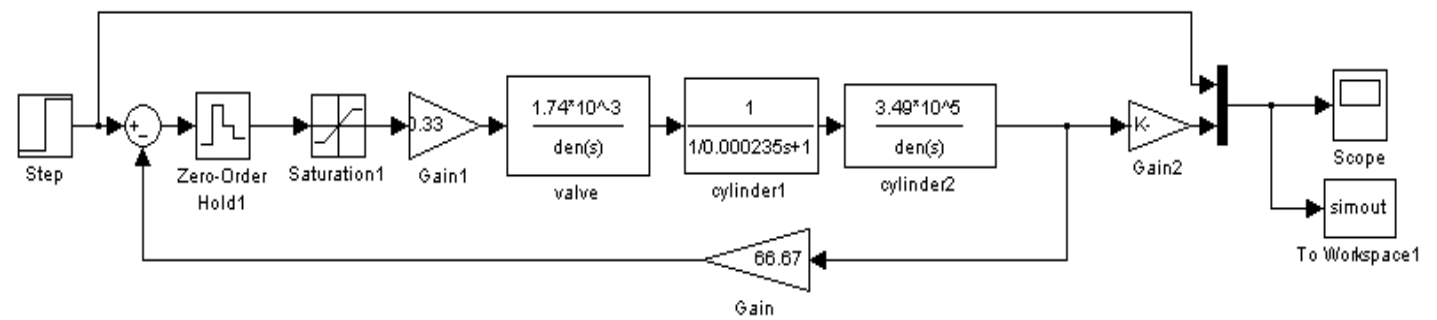

Fig. 5. $x_{v}>0$ The simulation block diagram of the system

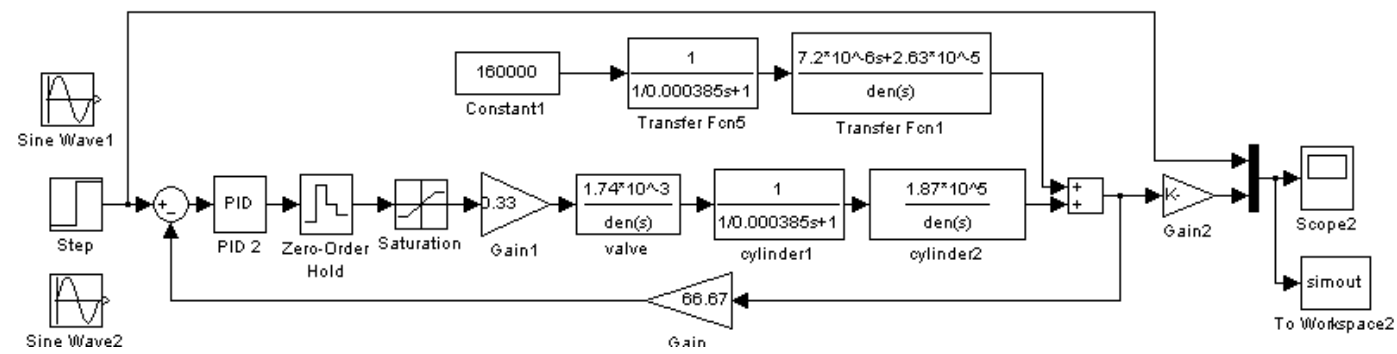

Fig. 6. $x_{v}<0$ The simulation block diagram of the system

We know that the response speed of the system is very fast with PID control. The rise time of the system is $0.02 \mathrm{~s}$, it meets the design requirement. 


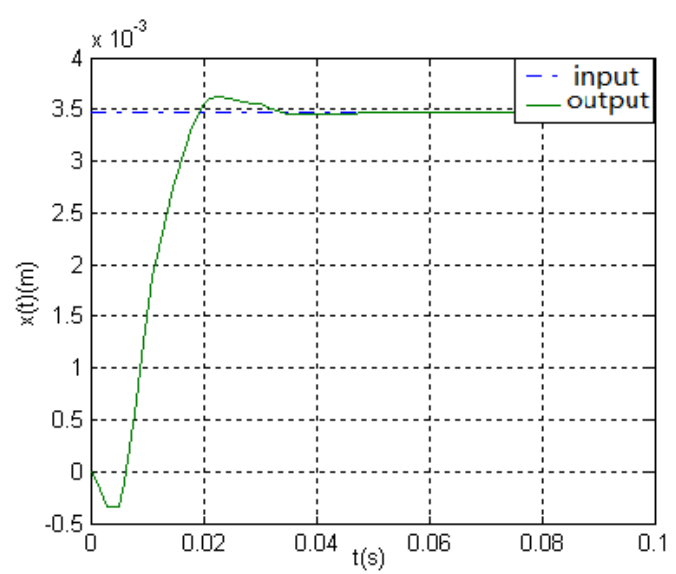

Fig. 7. $x_{v}>0$ Step response curve of system

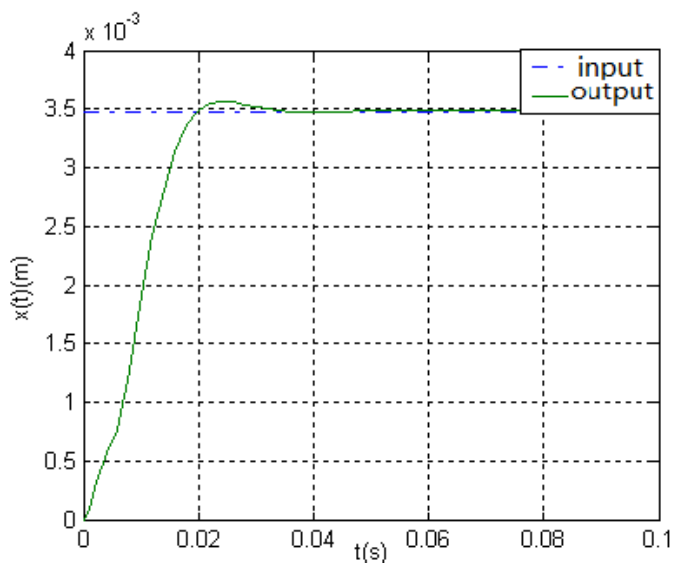

Fig. 8. $x_{v}<0$ Step response curve of system

\section{The simulation of PID control based on DSHplus}

The hydraulic system simulation diagram of PID control based on DSHplus is shown in Fig. 9.

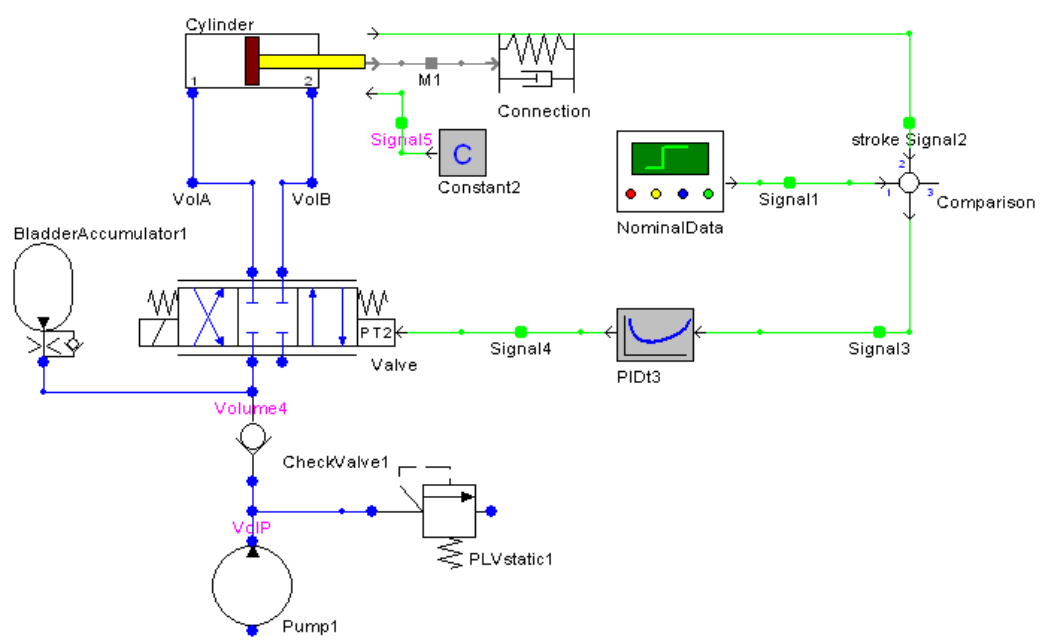

Fig. 9. The simulation diagram of Hydraulic system

The amplitude of phase step signal is $3.4733 \mathrm{~mm}$. The response curve of the system is shown in Fig. 10. From the step response curve of Fig. 10, we know that it has not obvious change to contrast

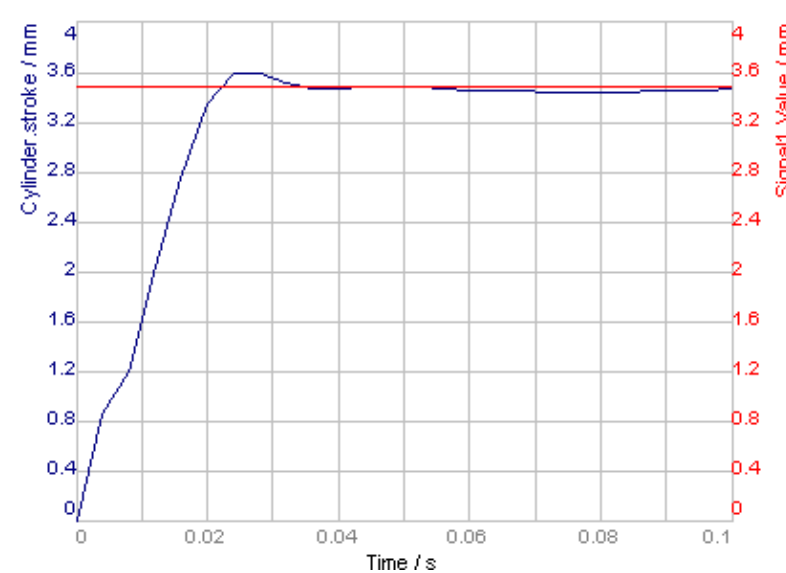

Fig. 10. Step response curve of system with the Fig. 8. The rise time of the system is $0.023 \mathrm{~s}$, it meets the frequency requirement of sine input system.

The amplitude of sine input signal is 41.8315 $\mathrm{mm}$, the frequency is $1 \mathrm{~Hz}$. The response curve of the system is shown in Fig. 11

The amplitude of sine input signal is 3.4733 $\mathrm{mm}$, the frequency is $5 \mathrm{~Hz}$. The response curve of the system is shown in Fig. 12。

From Fig. 11 and Fig. 12, we know that the tracking effect of the system is ideal, its error is in the allowed range. 


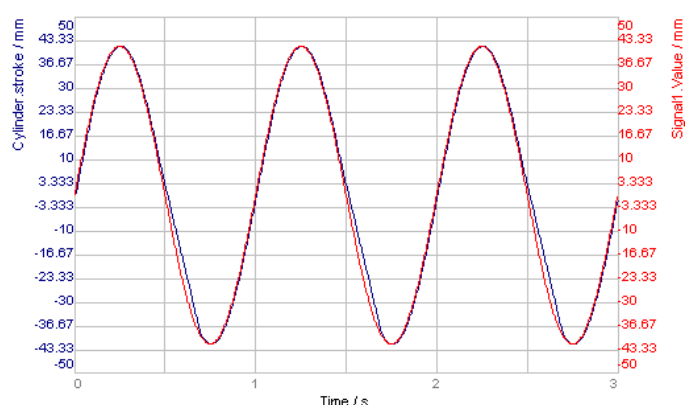

Fig. 11. Sinusoidal response curve of system

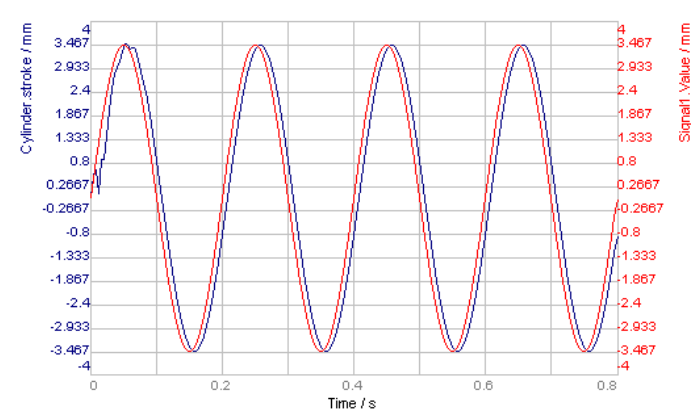

Fig. 12. Sinusoidal response curve of system

DSHplus establish graphical hydraulic simulation diagram to simulate and analyze, it has high efficiency in the simulation, and it is considering many nonlinear factors of hydraulic components and system. The simulation result is more close to the actual working condition of the system.

\section{Conclusions}

A new set of fatigue testing hydraulic system can be close to the actual conditions very well. Electro-hydraulic proportional closed-loop control system which fully integrated electrical and hydraulic advantages of both, the system have good control performance, big drive capacity. And the system is easy to be controlled by computer.

\section{References}

[1] Wei Ma, Deyu Li, Yuping Zhong, The development and use of bellows, J. Journal of Henan University of Science \& Technology. 2 (2004) 28-31.

[2] Huaxiang Wang, The life tests of elastic components, J. Instrument Manufacture. (1986) 12.

[3] Shenyang instrument institution 5 room $1^{\text {st }}$ group, The development situation of foreign metal bellows, J. Instrument Technology and Sensors. (1974) 1-7.

[4] Naining Huang, Research on Metal Bellows of Special Purpose, Shenyang, 2003.

[5] Yimin $\mathrm{Xu}$, The Analysis and Design of Electro-hydraulic Proportional Control System, Beijing, 2005. 